\title{
6D phase space electron beam analysis and machine sensitivity studies for ELI-NP GBS
}

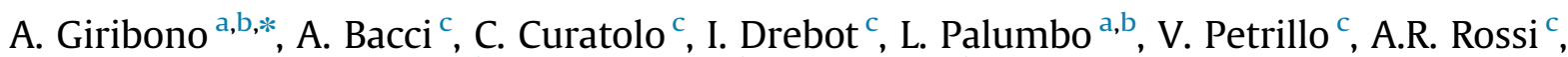 \\ L. Serafini ${ }^{\text {c }}$, C. Vaccarezza ${ }^{\mathrm{d}}$, A. Vannozzi ${ }^{\mathrm{a}}{ }^{\mathrm{b}}$, A. Variola ${ }^{\mathrm{d}}$ \\ a Dept. SBAI “La Sapienza” University, Via Antonio Scarpa,14 00161 Rome, Italy \\ b INFN-Roma1, Piazzale Aldo Moro,2 00161 Rome, Italy \\ ${ }^{\mathrm{C}}$ INFN-MI, Via Celoria 16, 20133 Milan, Italy \\ d INFN-LNF, Via Enrico Fermi,40 00044 Frascati Rome, Italy
}

\section{A R T I C L E I N F O}

\section{Article history:}

Received 14 November 2015

Received in revised form

15 February 2016

Accepted 15 February 2016

Available online 24 February 2016

Keywords:

Beam dynamics

Gamma source

Inverse Compton Scattering

\begin{abstract}
A B S T R A C T
The ELI-NP Gamma Beam Source (GBS) is now under construction in Magurele-Bucharest (RO). Here an advanced source of gamma photons with unprecedented specifications of brilliance $\left(>10^{21}\right)$, monochromaticity $(0.5 \%)$ and energy tunability $(0.2-19.5 \mathrm{MeV})$ is being built, based on Inverse Compton Scattering in the head-on configuration between an electron beam of maximum energy $750 \mathrm{MeV}$ and a high quality high power ps laser beam. These requirements make the ELI-NP GBS an advanced and challenging gamma ray source. The electron beam dynamics analysis and control regarding the machine sensitivity to the possible jitter and misalignments are presented. The effects on the beam quality are illustrated providing the basis for the alignment procedure and jitter tolerances.
\end{abstract}

(c) 2016 Elsevier B.V. All rights reserved.

\section{Introduction}

The ELI-NP Gamma Beam System (GBS) is a high spectral density and monochromatic $\gamma$ photon source based on the Inverse Compton Scattering phenomenon with unprecedented specifications of brilliance higher than $10^{21}$, r.m.s. bandwidth lower than $0.5 \%$ and energy tunability in the range $0.2-19.5 \mathrm{MeV}$. As pointed in detail in [1], to design a linac able to provide the requested electron beam quality the following factor of merit $\eta$, has to be maximized:

$\eta=\frac{Q_{b}}{\sigma_{t}^{2}\left[\left(\frac{\Delta \gamma}{\gamma}\right)^{2}+\left(\frac{\epsilon_{n}}{\sigma_{t}}\right)^{4}\right]^{\frac{1}{2}}}$

where $\epsilon_{n}$ is the transverse normalized emittance, $\sigma_{t}$ is the transverse spot size, $\Delta \gamma / \gamma$ is the energy spread and $Q_{b}$ is the charge of the electron beam at the interaction point (IP). In this form $\eta$ represents the $4 \mathrm{D}$ electron phase space density which scales like the spectral density of the emitted radiation (Eq. (3)-(5) in [1]), one of the main requirements of the ELI-NP $\gamma$-source listed in Table 1, and points out the relevance of electron beam emittance and energy spread contribution to the radiation quality. In the first section of this report the optimisation is described of the $250 \mathrm{pC}$ electron beam dynamics to guarantee the desired values for the electron beam emittance and

\footnotetext{
* Corresponding author.

E-mail address: anna.giribono@roma1.infn.it (A. Giribono).
}

energy spread; in the following the robustness of the linac design is addressed regarding machine element jitter and misalignments; on this basis alignment procedure and jitter tolerances are also provided.

\section{Electron beam dynamics}

On the above considerations an intense study has been performed to produce the final source design reported in ELI-NP official Technical Design Report (TDR) [2]; here the chosen electron beam parameters are: $\epsilon_{n}$ in the range $0.2-0.6 \mathrm{~mm}$ mrad, $\Delta \gamma$ $/ \gamma \leq 0.1 \%$ and energy tunability in the range $75-750 \mathrm{MeV}$. As reported in chapter 1 of [2] the behavior of the source spectral density as function of the electron beam spot size indicates $15 \mu$ $\mathrm{m} \leq \sigma_{t} \leq 30 \mu \mathrm{m}$ as the optimal range for the electron beam spot size at the interaction point with the best results for $\sigma_{t} \approx 20 \mu \mathrm{m}$.

To limit the normalized emittance dilution and energy spread of the electron beam, a hybrid scheme is adopted for the linac consisting in a SPARC-like S-band high brightness photoinjector [3] followed by a C-band RF linac as shown in Fig. 1 . The reason lies in having a bunch long enough, $\sigma_{z} \approx 1 \mathrm{~mm}$, in the RF gun to reduce the emittance degradation due to the space charge contribution, but taking advantage of the higher accelerating gradients provided by the C-band accelerating sections in the rest of the linac to compact its length. For the energy spread control a shorter bunch is needed instead in the C-band sections to avoid the RF curvature degradation effects, $\sigma_{z} \leq 280 \mu \mathrm{m}$, and this is obtained by means of the adopted 
velocity bunching scheme, resulting in a gentle longitudinal compression factor $<3$ at the photoinjector exit, as routinely and successfully applied at SPARC_LAB. More detail in the S-band injector, operating at $2.856 \mathrm{GHz}$, is composed of a 1.6 cell S-band RF gun equipped with a copper photo cathode and an emittance compensation solenoid, followed by two TW SLAC type S-band sections [4]. The RF gun accelerating field is $E_{a c c} \approx 120 \mathrm{MV} / \mathrm{m}$, while the two S-band structures operate at $22.5 \mathrm{MV} / \mathrm{m}$. The design electron beam parameters at the injector exit are: $\mathrm{E}=81.5 \mathrm{MeV}, \epsilon_{n}=$ $0.4 \mathrm{~mm}$ mrad, $\sigma_{t}=401 \mu \mathrm{m}, \Delta \mathrm{E} / \mathrm{E}=0.16 \%, \sigma_{z}=275 \mu \mathrm{m}$. The evolution along the injector of the electron beam normalized emittance $\left(\epsilon_{n}\right.$ red line), spot size ( $\sigma_{t}$ green dash-dot line) and longitudinal bunch length ( $\sigma_{z}$ blue dashed line) are plotted in Fig. 2. The downstream C-band RF linac operates at $5.712 \mathrm{GHz}$, with the accelerating structures designed and developed at LNF, [5], where the accelerating gradient can be set up to a maximum of $33 \mathrm{MV} / \mathrm{m}$, allowing enough margin for the off crest minimization of the energy spread in the 75$750 \mathrm{MeV}$ energy range $[1,6]$.

The $\mathrm{C}$ - band linac is divided in two main sections as shown in Fig. 1: the low energy one, composed of four accelerating sections, carries the electron beam up to the maximum energy $\mathrm{E}=320 \mathrm{MeV}$; $\mathrm{a}$ dogleg transport line downstream its exit, delivers the beam at the Low Energy Interaction Point (LE IP), avoiding in this way the bremsstrahlung radiation contribution. The downstream high energy linac is composed of eight accelerating sections and brings the electron beam up to the maximum energy $\mathrm{E}=750 \mathrm{MeV}$, then the electron beam, passing through a second dogleg beamline, reaches the High Energy Interaction Point (HE IP). In each of the two IP's regions a quadrupole triplet provides a flexible final focusing for matching the electron beam spot size vs the counter-propagating laser pulse.

The electron beam dynamics has been simulated for several Working Points (WPs) each corresponding to the user required $\gamma$ photon beam energy. The electron beam parameters listed in Table 2 result from the optimisation of the $250 \mathrm{pC}$ electron beam, composed of $30 \mathrm{~K}$ macro particles, tracked up to the IP: injector simulations are performed with the Astra code [7] able to take into account the space

Table 1

Summary of ELI-NP GBS specifications.

\begin{tabular}{lll}
\hline Energy & $0.2-19.5$ & $\mathrm{MeV}$ \\
Spectral density & $0.8-4.0 \cdot 10^{4}$ & $\mathrm{ph} / \mathrm{s} \cdot \mathrm{eV}$ \\
Peak brilliance & $10^{20}-10^{23}$ & $\mathrm{ph} / \mathrm{s} \cdot \mathrm{mm}^{2} \cdot \mathrm{mrad}^{2} \cdot 0.1 \%$ \\
Bandwidth [r.m.s.] & $\leq 0.5$ & $\%$ \\
Source size [r.m.s.] & $10-30$ & $\mu \mathrm{m}$ \\
$\begin{array}{c}\text { Pulse length [r.m.s] } \\
\text { \# photons/shot within FWHM }\end{array}$ & $0.7-1.5$ & $\mathrm{ps}$ \\
$\quad \leq 2.6 \cdot 10^{5}$ & \\
$\begin{array}{c}\text { \# photwidth } \\
\quad \text { bandwidth }\end{array}$ & $\leq 8.3 \cdot 10^{8}$ & \\
\hline
\end{tabular}

charge effects, relevant at very low energies; C-band RF linac simulations are performed with the Elegant code that includes the wakefields generated by the electron beam inside the accelerating structures together with the longitudinal space charge and the coherent and incoherent synchrotron radiation effects in the bending magnets. Looking at Table 2 results it has to be mentioned that for the very low energy $0.20 \mathrm{MeV}$ WP the energy spread turns out to be slightly out of specifications even if still acceptable by the user community. Accelerating gradients set in the range of $20-25 \mathrm{MV} / \mathrm{m}$ together with the proper off crest operation, i.e. dephasing of $\pm 15^{\circ}$ with respect to the maximum RF accelerating field, allow energy spread values of $0.08 \%$ at LE IP and of $0.04 \%$ at HE IP. The beam

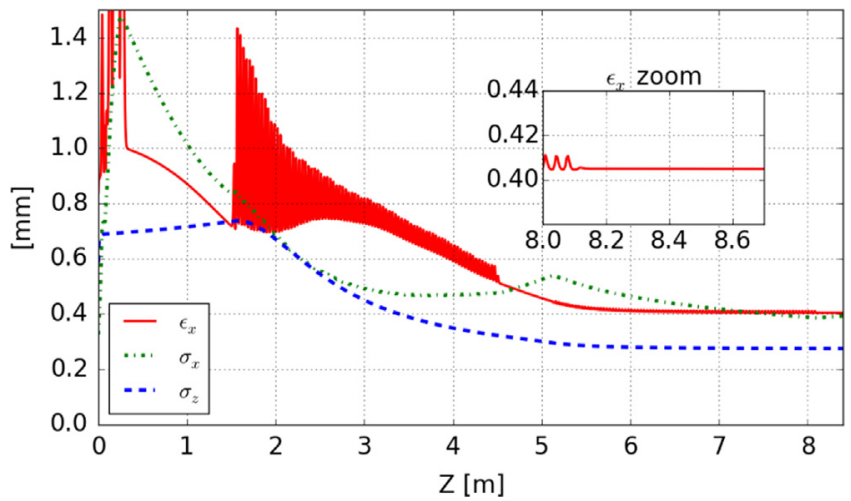

Fig. 2. Simulation results for the injector: evolution along the injector of the electron beam transverse normalized emittance ( $\epsilon$ red line), spot size ( $\sigma_{t}$ green dash-dot line) and longitudinal bunch length ( $\sigma_{z}$ blue dashed line) as obtained with the Astra code. (For interpretation of the references to color in this figure caption, the reader is referred to the web version of this paper.)

Table 2

Simulated parameters of the $250 \mathrm{pC}$ electron beam at low energy interaction point, LE IP, and high energy interaction point, HE IP. Following parameters result from the electron beam dynamics simulations.

\begin{tabular}{lllllllllll}
\hline $\begin{array}{l}\text { Interaction } \\
\text { point }\end{array}$ & LE IP & LE IP & LE IP & LE IP & LE IP & HE IP & HE IP & HE IP & \\
\hline $\begin{array}{c}\gamma \text { source } \\
\text { WP }\end{array}$ & 0.20 & 1.00 & 2.00 & 2.85 & 3.50 & 10.0 & 13.50 & 19.50 & $\mathrm{MeV}$ \\
$\begin{array}{c}\text { Energy } \\
\text { Energy } \\
\text { spread }\end{array}$ & 75 & 165 & 234 & 280 & 312 & 530 & 605 & 750 & $\mathrm{MeV}$ \\
$\begin{array}{c}\text { Bunch } \\
\text { length }\end{array}$ & 275 & 274 & 273 & 275 & 278 & 272 & 273 & 278 & $\mu \mathrm{m}$ \\
$\epsilon_{n} \quad 0.51$ & 0.44 & 0.44 & 0.45 & 0.41 & 0.44 & 0.44 & 0.41 & $\begin{array}{l}\mathrm{mm} \\
\mathrm{mrad}\end{array}$ \\
Spot size & 23.5 & 20.0 & 19.6 & 20.0 & 19.4 & 17.3 & 17.3 & 16.2 & $\mu \mathrm{m}$ \\
\hline
\end{tabular}

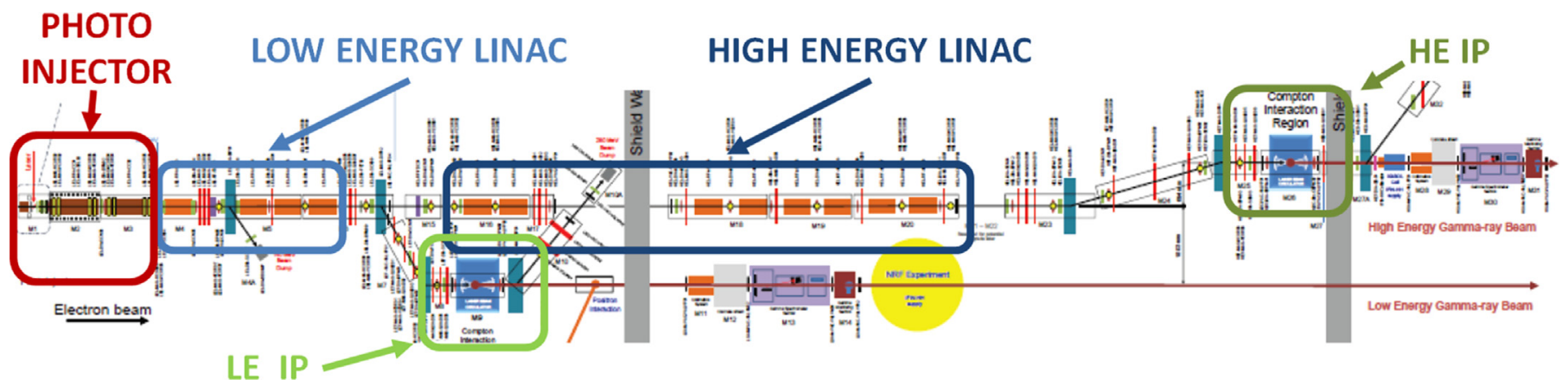

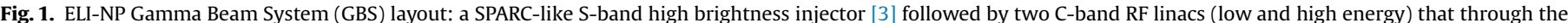
relative transfer lines provide the electron beam to the Low and High Energy Interaction Points (LE IP and HE IP) respectively [2]. 

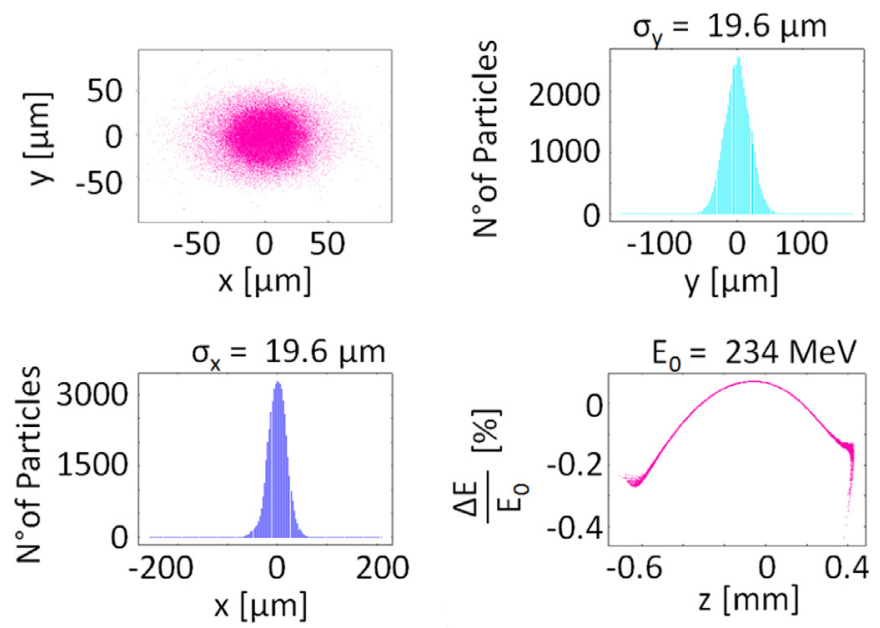

Fig. 3. Simulation results for the optimised $234 \mathrm{MeV}$ electron beam: simulated spot size and bunch length and simulated longitudinal phase space as obtained with Elegant tracking code.

transport line optics is matched to correct the horizontal dispersion at the dogleg exit. Furthermore, the final focusing system provides the spot size tunability in the range $18-25 \mu \mathrm{m}$ at the LE IP and in the range $10-25 \mu \mathrm{m}$ at the HE IP.

The simulation results are shown in Fig. 2 for the $234 \mathrm{MeV}$ electron beam required at the LE IP for the $2 \mathrm{MeV} \gamma$ photon beam production. The accelerating gradient is set at $23 \mathrm{MV} / \mathrm{m}$ with the following dephasing: $+15^{\circ}$ on the first two accelerating structures and $-3^{\circ}$ for the third and the fourth ones. The resulting electron beam parameters at the LE IP are: $\mathrm{E}=234 \mathrm{MeV}, \epsilon_{n}=0.44 \mathrm{~mm}$ mrad, $\sigma_{t}=19.6 \mu \mathrm{m}, \Delta \mathrm{E} / \mathrm{E}=0.082 \%, \sigma_{z}=273 \mu \mathrm{m}$. The obtained spot size and bunch length are reported in Fig. 3.

\section{Machine sensitivity studies}

The introduction of errors in the ELI-NP GBS electron beam lines affects the electron beam quality at the IP resulting in emittance and energy spread growth and large transverse trajectory errors [8]. Intense machine sensitivity studies have been performed in order to provide the basis for the alignment procedure and jitter tolerances. In order to ensure a reliable routine operation of the $\gamma$-photon source, starting from the accelerator main systems specifications in [2] and taking advantage of the experience acquired at SPARC _ LAB Thomson source [9], the maximum reasonable error values have been considered to face the most realistic situation, see Table 3 , trying not to count only on the best performance of the machine systems and to check the actual robustness of the source design.

The following results are related to the $250 \mathrm{pC}$ electron beam, composed of $30 \mathrm{~K}$ macroparticles, tracked up to the LE IP with a final energy of $280 \mathrm{MeV}$. Injector sensitivity studies have been carried out using Giotto [10] and Astra codes to provide tolerances and specifications for cathode laser system, power supplies and solenoids. Each bunch generated in the injector, has been tracked up to the LE IP along the C-band RF linac, whose sensitivity studies have been carried out using the Elegant code to provide tolerances and specifications for power supplies, magnetic elements and BPMs resolution. At first, the beam transport line is matched to obtain the desired electron beam parameters at the LE IP: $\epsilon_{n}=0.45 \mathrm{~mm} \operatorname{mrad}, \sigma_{t}=20 \mu \mathrm{m}, \Delta \mathrm{E} / \mathrm{E}=0.081 \%, \sigma_{z}=280 \mu \mathrm{m}$, then misalignments and jitter are introduced both in the injector and in the booster to perform the tracking.
Table 3

Maximum maximum error values for power supplies, magnetic elements and beam position monitors resolution.

\begin{tabular}{|c|c|c|}
\hline \multicolumn{3}{|l|}{ Injector } \\
\hline \multicolumn{3}{|l|}{ GUN } \\
\hline RF voltage $[\Delta \mathrm{V}]$ & \pm 0.2 & $\%$ \\
\hline RF phase $[\Delta \Phi]$ & \pm 0.2 & $\operatorname{deg}$ \\
\hline \multicolumn{3}{|l|}{ S-band accelerating sections } \\
\hline RF voltage $[\Delta \mathrm{V}]$ & \pm 0.2 & $\%$ \\
\hline RF phase $[\Delta \Phi]$ & \pm 0.2 & $\operatorname{deg}$ \\
\hline Alignment on transverse plane $[\Delta x y]$ & \pm 70 & $\mu \mathrm{m}$ \\
\hline \multicolumn{3}{|l|}{ Solenoids on GUN \& TW cavities } \\
\hline Alignment on transverse plane $[\Delta x y]$ & \pm 70 & $\mu \mathrm{m}$ \\
\hline \multicolumn{3}{|l|}{ Cathode laser system } \\
\hline Arrival time $[\Delta t]$ & \pm 200 & fs \\
\hline Pointing instabilities $[\Delta \mathrm{s}]$ & \pm 20 & $\mu \mathrm{m}$ \\
\hline Energy fluctuation & \pm 5 & $\%$ \\
\hline \multicolumn{3}{|l|}{ C-band RF linac } \\
\hline \multicolumn{3}{|l|}{ C-band accelerating sections } \\
\hline RF voltage $[\Delta \mathrm{V}]$ & \pm 0.2 & $\%$ \\
\hline RF phase $[\Delta \Phi]$ & \pm 1 & $\operatorname{deg}$ \\
\hline Alignment on transverse plane $[\Delta x y]$ & $\pm 70, \pm 100$ & $\mu \mathrm{m}$ \\
\hline \multicolumn{3}{|l|}{ Quadrupoles } \\
\hline Geometric strength $[\Delta \mathrm{k}]$ & \pm 0.3 & $\%$ \\
\hline Alignment on transverse plane $[\Delta x y]$ & $\pm 70, \pm 100$ & $\mu \mathrm{m}$ \\
\hline Tilt about incoming long. axis & \pm 1 & mrad \\
\hline \multicolumn{3}{|l|}{ Dipoles } \\
\hline Bend angle $[\Delta B]$ & \pm 0.1 & $\%$ \\
\hline Tilt about incoming long. axis & \pm 1 & mrad \\
\hline \multicolumn{3}{|l|}{ Steerers } \\
\hline Strength $\left[\Delta k_{S}\right]$ & \pm 0.2 & $\mu \mathrm{rad}$ \\
\hline \multicolumn{3}{|l|}{ Beam position monitors } \\
\hline Resolution & \pm 10 & $\mu \mathrm{m}$ \\
\hline
\end{tabular}

Machine sensitivity studies have been performed on samples of 100 machine runs each one obtained generating for each machine a tracking code input in which the elements errors are provided, in the chosen range, by means of the Matlab Latin Hypercube function that returns an n-by-p matrix, containing a latin hypercube sample of $n$ values (machine run identifier) on each of $p$ variables (element errors). For each column of matrix, the $\mathrm{n}$ values are randomly distributed with one from each interval $(0,1 / n),(1 / n, 2 / n), \ldots,(1-1 / n, 1)$, and they are randomly permuted, furthermore a normal random distribution of minus and plus sign is also applied. In this way the error matrix randomly factorize from $-100 \%$ to $+100 \%$ the considered error values listed in Table 3 for each element. With the aim to determine most dangerous error contributions, charge fluctuations in the range $237.5-262.5 \mathrm{pC}$, brought on by cathode laser energy jitter of $\pm 5 \%$, and errors in the C-band RF linac are considered one by one according to Table 3 . The trajectory correction in the booster is not applied in order to enhance the emittance dilution and large transverse trajectory errors due to misaligned accelerating structures and quadrupoles and to injection offset [8].

Injector sensitivity studies in case of charge fluctuations result in a maximum deviation from the optimised parameters at injector exit of $\pm 0.05 \%$ and do not cause significant linac optics mismatch in case of any other error. The electron beam affected by charge jitter has been injected in the RF linac. Tilt about the incoming longitudinal axis of $\pm 1^{\circ}$ such as jitter on dipole bending angle of $\Delta \mathrm{B}= \pm 0.1 \%$ and quadrupole strength of $\Delta \mathrm{k}= \pm 0.3 \%$ have negligible effects on the beam parameters at LE IP and so, are proposed, together with the cathode laser energy jitter, as specifications for the ELI-NP GBS machine. The accelerating structure voltage and phase jitter contributions mainly affect the energy spread of the electron beam. The occurrence of resultant energy spread values over the 100 machine runs is plotted in Fig. 4 in case of: $R F$ voltage jitter $(\Delta V$ red dashed line), RF phase jitter ( $\Delta \Phi$ blue dots), RF phase and voltage jitter (green line). Fig. 4 shows that the case of RF voltage jitter leads to almost all the machine runs producing an electron beam energy 


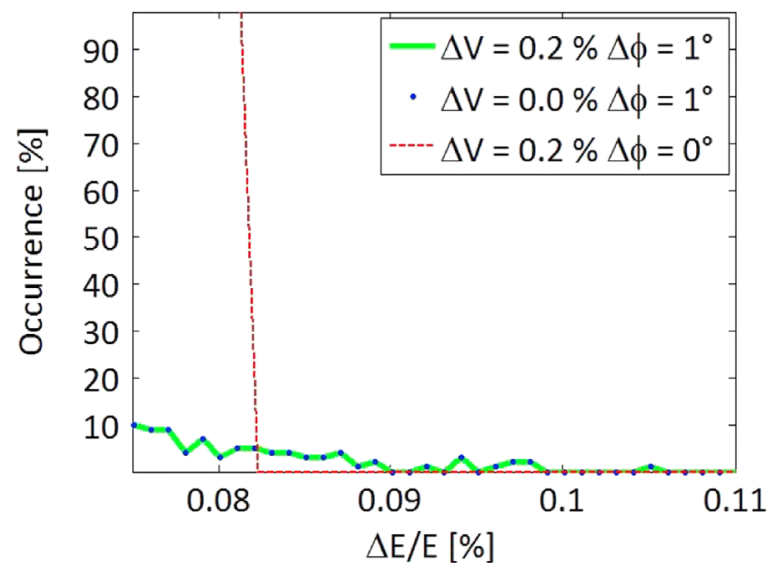

Fig. 4. Energy spread $(\Delta E)$ occurrence over 100 machine runs at LE IP in case of: RF voltage jitter (red dashed line), RF phase jitter (blue dots), RF phase and voltage jitter (green line). The analysis suggests that the energy spread can grow up to $0.1 \%$ if RF phase jitter in the range $\pm 1^{\circ}$ arises. (For interpretation of the references to color in this figure caption, the reader is referred to the web version of this paper.)

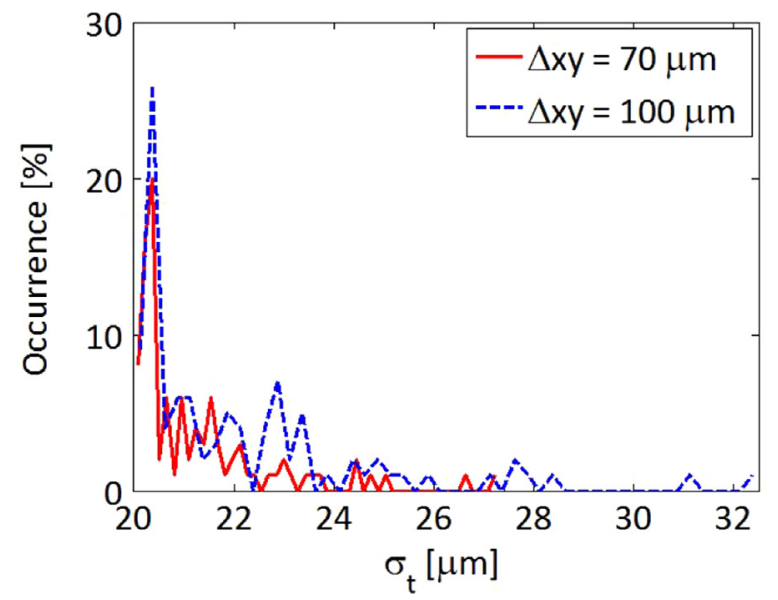

Fig. 5. Transverse spot size $\left(\sigma_{t}\right)$ occurrence over 100 machine runs at LE IP for $\Delta$ $x y= \pm 70 \mu \mathrm{m}$ (red line) and $\Delta x y= \pm 100 \mu \mathrm{m}$ (blue dashed line). The analysis suggests that the spot size can grow up to almost $33 \mu \mathrm{m}$ for $\Delta x y= \pm 100 \mu \mathrm{m}$. (For interpretation of the references to color in this figure caption, the reader is referred to the web version of this paper.)

spread of $\approx 0.081 \%$; nevertheless the case of RF phase jitter results in few machine runs producing an electron beam energy spread growth up to $\approx 0.1 \%$. The analysis suggests that energy spread lower than $0.1 \%$ needs RF phase jitter lower than $\pm 1^{\circ}$ in order to ensure a successful routinely operation of the $\gamma$-photon source. Misalignments on transverse plane, $\Delta x y$, are considered on both accelerating structures and quadrupoles in the range $\pm 70 \mu \mathrm{m}$ or $\pm 100 \mu \mathrm{m}$. While the energy spread remains always lower than $0.088 \%$, the normalized emittance and the spot size grow up to respectively $0.7 \mathrm{~mm}$ mrad and almost $33 \mu \mathrm{m}$ for $\Delta x y= \pm 100 \mu \mathrm{m}$. More relaxed is the scenario for $\Delta x y= \pm 70 \mu \mathrm{m}$ where $\epsilon_{n}$ is lower than $0.6 \mathrm{~mm}$ mrad and $\sigma_{t}$ is lower than $27 \mu \mathrm{m}$. Fig. 5 shows the occurrence of resultant $\sigma_{t}$ values over the 100 machine runs for $\Delta x y= \pm 70 \mu \mathrm{m}$ (red line) and $\Delta x y= \pm 100 \mu \mathrm{m}$ (blue dashed line). Even if the case of $\Delta x y= \pm 100 \mu \mathrm{m}$ results in few machine runs producing a $\sigma_{t}$ higher than $30 \mu \mathrm{m}$, the proposed alignment precision is at least of $\pm 70 \mu \mathrm{m}$.

According to previous study results and to Table 3, an enlarged sample of 352 machine runs is considered introducing also errors in the injector. The 352 machine runs are generated with the same procedure used before, but now misalignments are established in the range $\pm 70 \mu \mathrm{m}$. Once more, injector sensitivity studies show negligible effects on the beam parameters at the injector exit not affecting the C-band RF linac matching. At this point the trajectory correction is applied to control the large transverse trajectory errors due to misaligned accelerating structures and quadrupoles, including in the Elegant code the steerer strength jitter and BPM resolution as listed in Table 3. Steerers are switched on centering the beam on all BPMs, avoiding off-axis trajectories, and on the two cavity BPMs placed at the entrance and exit of the interaction region, to maximize the source luminosity. (The alignment errors on steerer magnets and BPMs have not been introduced so far and it will be done next). The result of the trajectory correction, that allows us to manage injection offset and the spot size growth due to emittance dilution, is an electron beam with $\epsilon_{n}=(0.45 \pm 0.03) \mathrm{mm}$ mrad, $\sigma_{t}=(22.5 \pm 1.5) \mu \mathrm{m}$ and $\Delta \mathrm{E} / \mathrm{E}=(0.081 \pm 0.005) \%$, confirming the robustness of the source to jitter and misalignments inside the tolerance range listed in Table 3.

\section{Conclusions}

The optimisation work on the electron beam dynamics of the ELI-NP Gamma Beam System has been described regarding machine sensitivity studies aiming to check the robustness of the source in terms of jitter and misalignments. A tolerance range has been indicated in Table. Table 3 and the obtained simulations results have been presented satisfactorily close to the nominal machine parameters. Other type of deviations as steerer magnets and BPM alignment errors will be considered next to complete the analysis towards the multibunch operation sensitivity studies.

\section{References}

[1] A. Bacci, D. Alesini, P. Antici, M. Bellaveglia, R Boni, E Chiadroni, A Cianchi, C Curatolo, G. Di Pirro, A. Esposito, M. Ferrario, A. Gallo, G. Gatti, A. Ghigo, M. Migliorati, A. Mostacci, L. Palumbo, V. Petrillo, R. Pompili, C. Ronsivalle, A.R. Rossi, L. Serafini, B. Spataro, P. Tomassini, C. Vaccarezza, Electron linac design to drive bright compton back-scattering gamma-ray sources, J. Appl. Phys. 113 (19), 2013, http://dx.doi.org/10.1063/1.4805071. 〈http://scitation.aip.org/con tent/aip/journal/jap/113/19/10.1063/1.4805071>.

[2] L. Serafini, et al., Technical Design Report: Eurogammas Proposal for the ELINP Gamma Beam System, Scientific Editor L. Serafini.

[3] M. Ferrario, D. Alesini, A. Bacci, M. Bellaveglia, R. Boni, M. Boscolo, M. Castellano, E. Chiadroni, A. Cianchi, L. Cultrera, G. Di Pirro, L. Ficcadenti, D. Filippetto, V. Fusco, A. Gallo, G. Gatti, L. Giannessi, M. Labat, B. Marchetti, C. Marrelli, M. Migliorati, A. Mostacci, E. Pace, L. Palumbo, M. Quattromini, C. Ronsivalle, A.R. Rossi, J. Rosenzweig, L. Serafini, M. Serluca, B. Spataro, C. Vaccarezza, C. Vicario, Phys. Rev. Lett. 104 (2010) 054801, http://dx.doi.org/ 10.1103/PhysRevLett.104.054801, URL 〈http://link.aps.org/doi/10.1103/PhysRev Lett.104.054801).

[4] R. Neal, General Editor, The Stanford Two-mile Accelerator, Benjamin, W.A., Inc., New York.

[5] D. Alesini, S. Bini, R. Di Raddo, V. Lollo, L. Pellegrino, L. Ficcadenti, V. Pettinacci, L. Palumbo, L. Serafini, Design and RF test of damped c-band accelerating structures for the ELI-NP linac, Proceedings of 5th International Particle Accelerator Conference (IPAC 2014), THPRI042, 2014, 〈http://dx.doi.org/http:// jacow.org/IPAC2014/papers/thpri042.pdf $\rangle$.

[6] C. Vaccarezza, D. Alesini, A. Bacci, M. Bellaveglia, R. Boni, E. Chiadroni, A Cianchi, G. Di Pirro, I. Drebot, M. Ferrario, A Gallo, G. Gatti, A Ghigo, A. Giribono, A. Mostacci, D. Palmer, L. Palumbo, V. Petrillo, C. Ronsivalle, A. R. Rossi, L. Serafini, B. Spataro, P. Tomassini, Optimizing RF linacs as drivers for inverse compton sources: the ELI-NP case, In: Proceedings of LINAC14, 2014.

[7] K. Floetmann, Astra, 〈http://www.desy.de/ mpyflo/Astra_dokumentationhttp://desy.de/ mpyflo/Astra_dokumentation $\rangle$.

[8] A. Mosnier, Instabilities in linacs, CAS - CERN Accelerator School: 5th Advanced Accelerator Physics Course.

[9] C. Vaccarezza, D. Alesini, M. Anania, A. Bacci, M. Bellaveglia, P. Cardarelli, E. Chiadroni, A. Cianchi, C. Curatolo, P. Delogu, G. Di Domenico, D. Di Giovenale, G. Di Pirro, M. Ferrario, F. Filippi, A. Gallo, M. Gambaccini, G. Gatti, A. Giribono, B. Golosio, A. Mostacci, P. Oliva, D. Palmer, V. Petrillo, R. Pompili, S. Romeo, A Rossi, L. Serafini, P. Tomassini, F. Villa, The SPARC_LAB Thomson Source Commissioning, Proceedings of 5th International Particle Accelerator Conference (IPAC 2014), MOPRO078, 2014, 〈http://jacow.org/IPAC2014/papers/ mopro078.pdf $\rangle$.

[10] A. Bacci, C. Maroli, V. Petrillo, A. Rossi, L. Serafini, Maximizing the brightness of an electron beam by means of a genetic algorithm, Nucl. Instrum. Methods Phys. Res. B, 263, 2007, http://dx.doi.org/10.1016/j.nimb.2007.07.006. 